\title{
Thermal and fast neutron detection in chemical vapor deposition single-crystal diamond detectors
}

\author{
S. Almaviva, ${ }^{1}$ M. Marinelli, ${ }^{1}$ E. Milani ${ }^{1}$ G. Prestopino, ${ }^{1}$ A. Tucciarone, ${ }^{1}$ C. Verona,${ }^{1}$ \\ G. Verona-Rinati, ${ }^{1, a)}$ M. Angelone, ${ }^{2}$ D. Lattanzi, ${ }^{2}$ M. Pillon, ${ }^{2}$ R. M. Montereali, ${ }^{3}$ and \\ M. A. Vincenti ${ }^{3}$ \\ ${ }^{1}$ Dipartimento di Ingegneria Meccanica, Università di Roma "Tor Vergata," Via del Politecnico 1, \\ I-00133 Roma, Italy \\ ${ }^{2}$ Associazione EURATOM-ENEA sulla Fusione, Via E. Fermi 45, I-00044 Frascati (Roma), Italy \\ ${ }^{3}$ ENEA-FIS C.R. Frascati, Via E. Fermi 45, I-00044 Frascati (Roma), Italy
}

(Received 29 September 2007; accepted 29 November 2007; published online 3 March 2008)

\begin{abstract}
Recently, a compact solid-state neutron detector capable of simultaneously detecting thermal and fast neutrons was proposed [M. Marinelli et al., Appl. Phys. Lett. 89, 143509 (2006)]. Its design is based on a $p$-type/intrinsic/metal layered structure obtained by Microwave Plasma Chemical Vapor Deposition (CVD) of homoepitaxial diamond followed by thermal evaporation of an $\mathrm{Al}$ contact and a ${ }^{6} \mathrm{LiF}$ converting layer. Fast neutrons are directly detected in the CVD diamond bulk, since they have enough energy to produce the ${ }^{12} \mathrm{C}(n, \alpha){ }^{9} \mathrm{Be}$ reaction in diamond. Thermal neutrons are instead converted into charged particles in the ${ }^{6} \mathrm{LiF}$ layer through the ${ }^{6} \mathrm{Li}(n, \alpha) T$ nuclear reaction. These charged particles are then detected in the diamond layer. The thickness of the ${ }^{6} \mathrm{LiF}$ converting layer and the CVD diamond sensing layer affect the counting efficiency and energy resolution of the detector both for low- (thermal) and high-energy neutrons. An analysis is carried out on the dynamics of the ${ }^{6} \mathrm{Li}(n, \alpha) T$ and the ${ }^{12} \mathrm{C}(n, \alpha){ }^{9} \mathrm{Be}$ reactions products, and the distribution of the energy released inside the sensitive layer is calculated. The detector counting efficiency and energy resolution were accordingly derived as a function of the thickness of the ${ }^{6} \mathrm{LiF}$ and CVD diamond layers, both for thermal and fast neutrons, thus allowing us to choose the optimum detector design for any particular application. Comparison with experimental results is also reported. (C) 2008 American Institute of Physics. [DOI: 10.1063/1.2838208]
\end{abstract}

\section{INTRODUCTION}

Neutron detection is obviously a primary issue when dealing with nuclear reactors, either fusion or fission ones. The ideal neutron detector should be compact (to allow its optimal positioning), fissile-material-free (for security reasons and ease of operation), and radiation- and temperatureresistant (to allow operation in harsh environments, such as in proximity to the reactor core). In addition, it should be sensitive to both thermal and fast neutrons. Traditionally used neutron detectors (fission chambers, gas counters, silicon detectors) fail in at least one of these requirements.

Although it is well known that a compact solid-state detector based on an inert and radiation-resistant material can be realized using very pure diamond, ${ }^{1-5}$ only very recently has the fabrication of Chemical Vapor Deposition (CVD) diamond neutron detectors in a $p$-type/intrinsic/metal layered configuration with highly reproducible characteristics been reported. ${ }^{5,6} \alpha$-particle irradiation tests have shown $100 \%$ charge collection efficiency and approximately $1 \%$ energy resolution, while neutron radiation hardness up to at least 2 $\times 10^{14} \mathrm{n} / \mathrm{cm}^{2}$ fast $(14 \mathrm{MeV})$ neutron fluence was confirmed to date.

In such devices, fast neutrons are detected directly in the bulk of the intrinsic diamond layer through the ${ }^{12} \mathrm{C}(n, \alpha){ }^{9} \mathrm{Be}$ reaction. The produced ${ }^{9} \mathrm{Be}+\alpha$ ions have a total energy

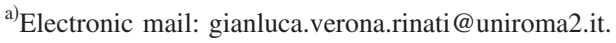

$E_{\alpha+\mathrm{Be}}=E_{n}-5.7 \mathrm{MeV}$, where $E_{n}$ is the energy of the impinging neutron (14.8 MeV in this test). These ionizing particles have enough energy to produce a measurable signal even in a few- $\mu$ m-thick detector $(9.1 \mathrm{MeV}$ in this test).

Sensitivity to thermal neutrons is achieved by depositing a $95 \%$ enriched ${ }^{6} \mathrm{LiF}$ layer on top of the upper (positively biased) $\mathrm{Al}$ contact. The ${ }^{6} \mathrm{LiF}$ layer acts as a converting material from thermal neutron to highly ionizing particles through the nuclear reaction ${ }^{6} \operatorname{Li}(n, \alpha) T$. Tritium ions and $\alpha$-particles are emitted in opposite directions, so that either of them, but not both, are detected. Their energies are 2.73 and $2.07 \mathrm{MeV}$, respectively.

Clearly, the detection process of thermal neutrons is affected by the ${ }^{6} \mathrm{LiF}$ layer thickness: the thicker the ${ }^{6} \mathrm{LiF}$ layer, the higher the conversion efficiency and the counting rate. On the other hand, tritium ions and the $\alpha$-particles lose part of their energy in the ${ }^{6} \mathrm{LiF}$ layer, so that optimal energy resolution calls for thin layers. In the case of fast neutrons, which are directly detected in the active diamond layer, the thickness of this latter layer is obviously decisive for the best counting rate and energy resolution.

In this article, we report on a thorough examination of the conversion reactions dynamics and the effects of the ${ }^{6} \mathrm{LiF}$ and CVD diamond layer thickness on the detected energy distribution. This will allow us to choose, for any given application, the best ${ }^{6} \mathrm{LiF}$ and $\mathrm{CVD}$ diamond layer thickness in 


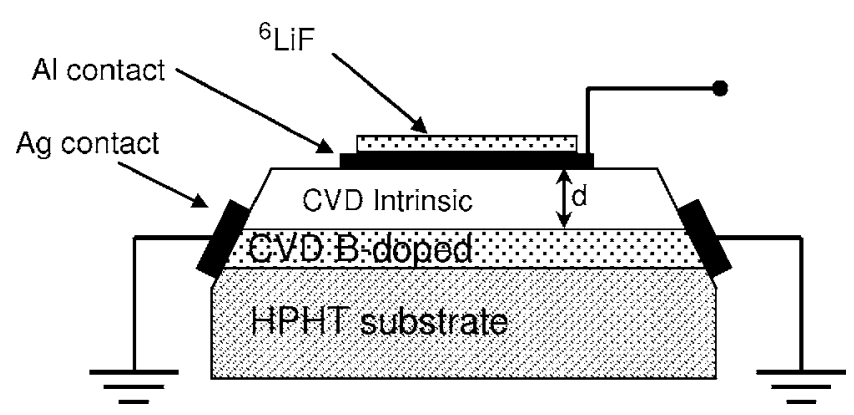

FIG. 1. Scheme of the device configuration.

the trade-off between counting rate and energy resolution for both fast and thermal neutrons. Comparison with experimental results is also reported.

\section{EXPERIMENTAL}

The neutron detectors used in this experiment were fabricated from CVD diamond films, according to the procedure described below.

As reported in Ref. 5, many of these detectors were realized, all showing very similar detection properties. In particular, for all of them the pulse height analysis (PHA) spectrum measured under 5.5 $\mathrm{MeV}{ }^{241} \mathrm{Am} \alpha$-particle irradiation showed a single peak corresponding to $100 \%$ collection efficiency and $0.5 \%-2 \%$ energy resolution. No polarization or other memory effects were observed.

Commercial high-pressure-high-temperature (HPHT) Ib single-crystal diamonds were used as substrates, having a square shape with about $3.5 \mathrm{~mm}$ side length and approximately $400 \mu \mathrm{m}$ thickness. A two-step homoepitaxial CVD process was used to realize each detector. A-15 $\mu$ m-thick boron-doped diamond buffer layer with approximately $5 \Omega \mathrm{cm}$ resistivity was first deposited onto the HPHT substrates, followed by an intrinsic diamond film deposition in the thickness range $25-100 \mu \mathrm{m}$. This second step was made in a completely separate CVD reactor, in order to avoid unintentional boron contamination of the intrinsic layer. After the intrinsic diamond layer deposition, the diamond surface was oxidized by thermal annealing in air at $500{ }^{\circ} \mathrm{C}$. The detectors were then realized using the conductive B-doped layer as a backing contact, while a 2.5-mm-diam, 100-nmthick $\mathrm{Al}$ contact is thermally evaporated on the intrinsic growth surface. This multilayer geometry allows us to separate the response of the high-quality intrinsic CVD sensing layer from the one arising from the HPHT substrate, if any, with no need to mechanically remove it. The detection signal only comes from the CVD layer where the electric field is confined.

Finally, the ${ }^{6} \mathrm{LiF}$ layer is deposited on the $\mathrm{Al}$ contact. All measurements were repeated for three different ${ }^{6} \mathrm{LiF}$ layer thickness values $(0.5,1.6$, and $3.0 \mu \mathrm{m}$, respectively).

Figure 1 shows a sketch of the device. The detector bias used in the experiments is $+5 \mathrm{~V} / \mu \mathrm{m}$, with positive voltage applied to the Al contact. Negative polarization of the devices always gives rise to useless noisy measurements, due to a high current from the boron-doped diamond contact.

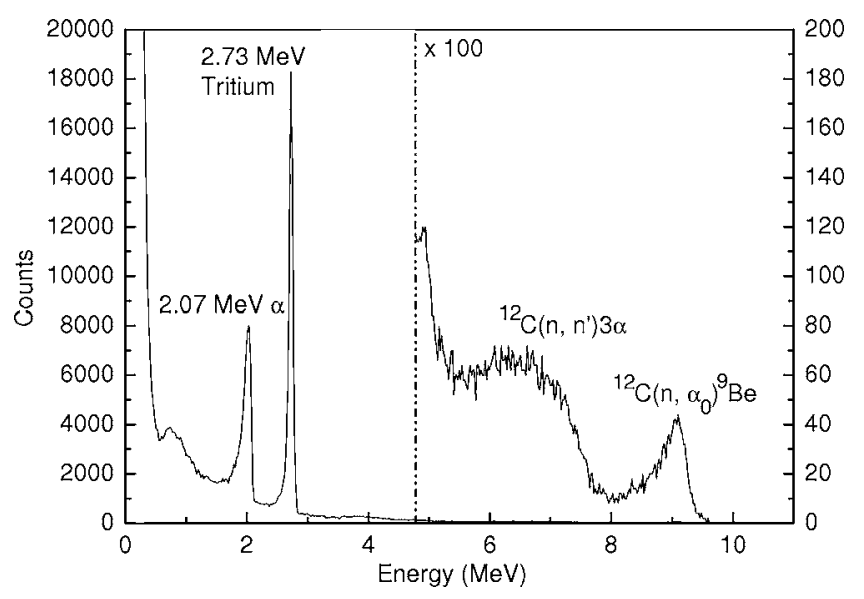

FIG. 2. PHA spectrum under neutron irradiation. In the right side of the figure, the vertical scale is magnified by a factor 100 .

The charge-sensitive electronic chain was calibrated by using a $100 \%$ collection efficiency Si detector, and the measured charge was related to the energy detected by diamond assuming $13.2 \mathrm{eV}$ as the average energy for creating an electron-hole pair. ${ }^{2}$

During the neutron detection tests, the devices were connected to an electronic chain composed by an Ortec $142 \mathrm{~A}$ charge preamplifier, a $0.5 \mu$ s shaping amplifier, and a multichannel analyzer. Neutron irradiations were performed at the Frascati Neutron Generator ${ }^{7}$ (FNG) with $14.8 \mathrm{MeV}$ neutrons produced by the $260 \mathrm{keV}$ deuteron beam impinging on a tritiated target. Neutrons are produced via the $T(d, n)^{4} \mathrm{He}$ reaction. Thermal neutrons where obtained by slowing down the fast neutrons through a cylindrical polymethylmethacrylate (PMMA) moderator with a radius of $10 \mathrm{~cm}$. The diamond-LiF detector was placed in a vertical hole located in the center of the moderator. Due to the relatively small thickness of the moderator, only a fraction of the incident neutrons is slowed down. A Monte Carlo neutron transport simulation showed that only about $10 \%$ of incident neutrons are in the thermal range. 5

\section{RESULTS AND DISCUSSION}

In Fig. 2 the PHA spectrum of a diamond detector under neutron irradiation is reported. In this case, the thinner (about $0.5 \mu \mathrm{m}){ }^{6} \mathrm{LiF}$ layer was used. Above the low-energy background, the two sharp peaks at about 2 and $2.7 \mathrm{MeV}$ are due to $\alpha$-particles and tritium ions, respectively. At higher energies (note the $\times 100$ amplified intensity scale on the right), the broader peak at about $9.1 \mathrm{MeV}$ is due to the ${ }^{12} \mathrm{C}(n, \alpha){ }^{9} \mathrm{Be}$ reaction in diamond. Despite the small thickness of the CVD layer (approximately $25 \mu \mathrm{m}$ ), the $\mathrm{Be}+\alpha$ ion peak is clearly visible and well separated from the ${ }^{12} \mathrm{C}\left(n, n^{\prime}\right) 3 \alpha$ continuum at lower energies.

Since the thermal cross section ${ }^{6} \operatorname{Li}(n, \alpha) T$ is four orders of magnitude higher than those of the other competing reactions occurring both in lithium and carbon, ${ }^{8}$ in the spectrum reported in Fig. 2 the peaks due to thermal neutron detection are much more intense than those due to fast neutrons, even 
using a very thin ${ }^{6} \mathrm{LiF}$ layer. Nevertheless, it is clear that the detector is fully capable of simultaneously detecting thermal and fast neutrons.

In the following, we will separately discuss the influence of the ${ }^{6} \mathrm{LiF}$ layer and the CVD diamond active thickness on the detection of thermal and energetic neutrons.

\section{A. ${ }^{6}$ LiF layer thickness measure}

For a correct evaluation of the device operation, the exact ${ }^{6} \mathrm{LiF}$ layer thickness must be known. Since we have no in situ characterization of the ${ }^{6} \mathrm{LiF}$ layer thickness during deposition, we have measured it after deposition. To do this, a collimated beam of $5.5 \mathrm{MeV} \alpha$-particles was directed onto the detector, varying the angle $\theta$ between the beam direction and the normal to the detector surface. Clearly, the energy lost by $\alpha$-particles in the ${ }^{6} \mathrm{LiF}$ layer increases with increasing $\theta$ (for a given layer thickness). The energy of the $\alpha$-particles, as measured by the detector, was then calculated as follows. If the path traveled by $5.5 \mathrm{MeV} \alpha$-particles in $\mathrm{LiF}$ is lower than approximately $12 \mu \mathrm{m}$, the relative Bragg curve can be well approximated by an exponential function,

$$
-\frac{d E}{d x}=A e^{B x} .
$$

The two parameters $A$ and $B$ were derived by the best fit of Eq. (1) with the Bragg curve of $5.5 \mathrm{MeV} \alpha$-particles in $\mathrm{LiF}$ as calculated by a standard nuclear simulation program SRIM. $^{9}$

The energy of the $\alpha$-particle after traveling a distance $x$ in the LiF layer is then obtained by integrating Eq. (1),

$$
E(x)=E_{0}-\frac{A}{B}\left(e^{B x}-1\right),
$$

where $E_{0}$ is the starting energy of the $\alpha$-particle. By neglecting straggling effects, the energy of the $\alpha$-particles as measured by the detector is finally given by

$$
E(\theta)=E_{0}-\frac{A}{B}\left(e^{B d / \cos \theta}-1\right) .
$$

Fitting the measured $\alpha$-particle energies with this formula allows a precise calculation of the ${ }^{6} \mathrm{LiF}$-layer thickness. As an example, in Fig. 3 the energies versus $\theta$ experimental data are reported together with the best fit of Eq. (3) for one of the three ${ }^{6} \mathrm{LiF}$ layers used. In this case, the calculated thickness is $1.60 \pm 0.05 \mu \mathrm{m}$, to be compared with the $2.2 \mu \mathrm{m}$ value simply estimated from the deposition time. The same procedure applied in the other two cases leads to $3.0 \pm 0.1$ and $0.45 \pm 0.05 \mu \mathrm{m}$, respectively.

\section{B. Thermal neutrons}

The thickness of the diamond active layer plays no role as long as it is larger than the penetration depth of $2.73 \mathrm{MeV}$ tritium ions and $2.07 \mathrm{MeV} \alpha$-particles (about 21 and $3.7 \mu \mathrm{m}$, respectively). This condition is always fulfilled in our case and therefore only the ${ }^{6} \mathrm{LiF}-$ layer thickness rules the conversion efficiency of thermal neutrons into detectable particles, thus the detector counting efficiency for thermal

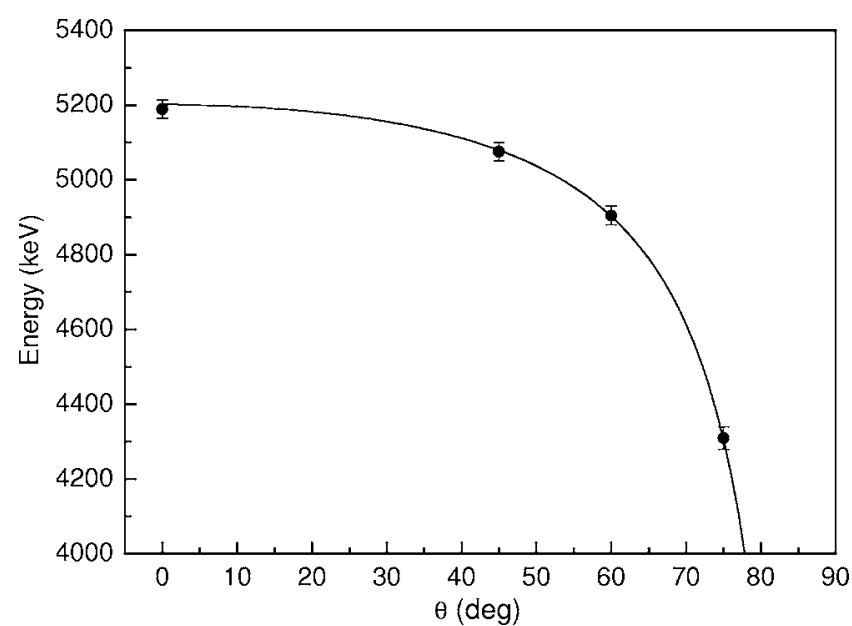

FIG. 3. Energy measured by the detector covered by $1.6-\mu \mathrm{m}$-thick ${ }^{6} \mathrm{LiF}$ (full circle) as a function of the angle of incidence under ${ }^{241} \mathrm{Am} \alpha$-particle irradiation. The full line is the best fit used to determine the ${ }^{6} \mathrm{LiF}$ thickness.

neutrons. At the same time, the produced charged particles lose a small part of their energy in the ${ }^{6} \mathrm{LiF}$ layer, which produces an energy spread, reducing the energy resolution of the detector. This energy spread can be calculated as discussed below and the computed energy resolution can be compared to the experimental one.

The procedure used to simulate the PHA spectrum of the detector is as follows. The Bragg curves of $2.07 \mathrm{MeV}$ alphas and $2.73 \mathrm{MeV}$ tritium ions in $\mathrm{LiF}$ were approximated to the empirical function,

$$
-\frac{d E}{d x}=\frac{A e^{B x}}{1+e^{x-C / D}} .
$$

The four parameters $A, B, C$, and $D$ were derived for either $\alpha$-particles or tritium ions by the best fit of Eq. (4) to the Bragg curves as calculated by the nuclear simulation program SRIM. Equation (4) is Eq. (1) multiplied by a Fermi function in order to describe also the cutoff region of the Bragg distribution, which was not necessary in the preceding section. Monte Carlo simulation of the released energy spectra was then performed by randomly generating $\alpha$-particles or tritium ions inside the ${ }^{6} \mathrm{LiF}$ layer with a homogeneous distribution of the creation point and isotropic distribution of emission angle.

In the simulation, the path of $\alpha$ 's and tritons is assumed to be a straight line. This approximation is certainly valid close to the generation point of the particles, but the motion of the particles becomes more complex at low energy, i.e., close to the end of the particle path. However, this can affect only $\alpha$-particles and tritons stopping very close to the diamond-LiF separation surface, thus releasing inside diamond only a small fraction of their starting energy. For this reason, only the very low-energy part of the spectrum, usually not experimentally accessible due to the presence of noise and other low-energy reaction, can be affected by the adopted approximation.

In Fig. 4, the results of the simulations are shown (dotted lines) and compared with the experimental PHA spectra (full lines) for the three ${ }^{6} \mathrm{LiF}$ layers used. The only adjustable parameter in the calculated PHA spectra is the amplitude, 


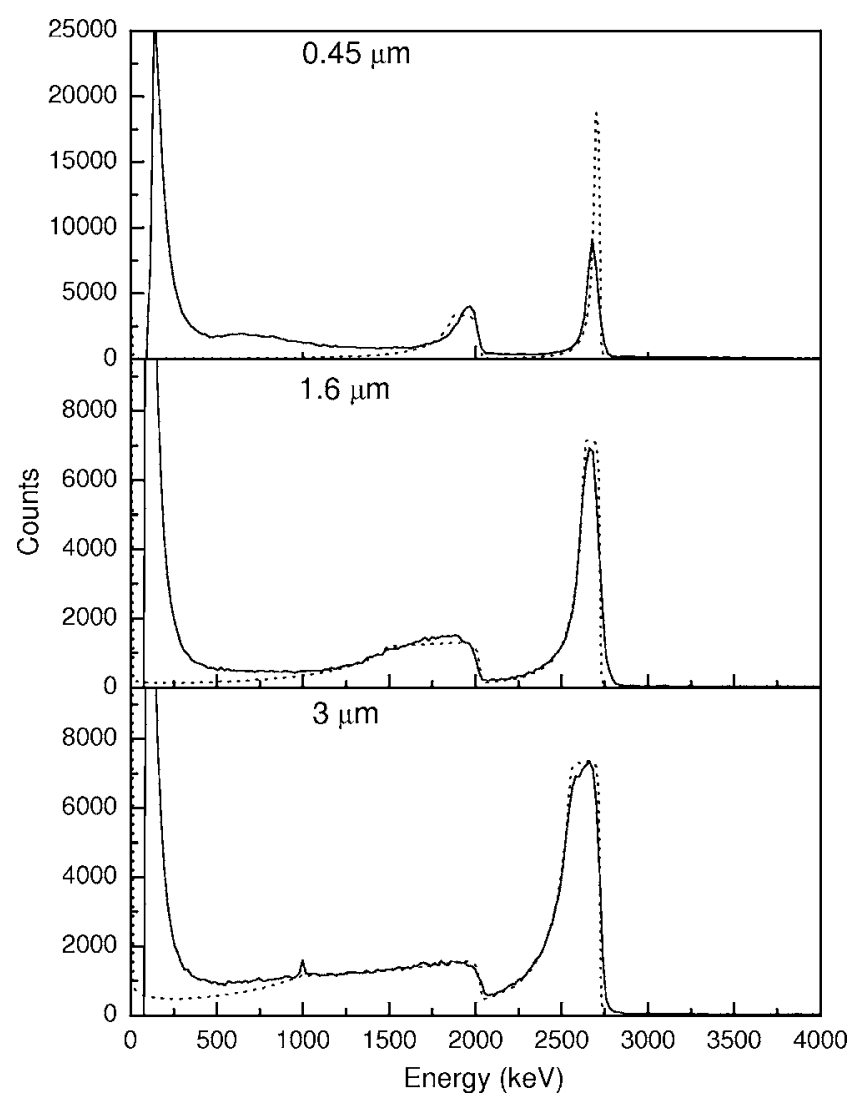

FIG. 4. PHA experimental spectra (full lines) under thermal neutron irradiation for three different ${ }^{6} \mathrm{LiF}$-layer thicknesses as compared with numerical simulations (dashed lines).

which has been chosen in such a way to have a good correspondence between calculated and experimental $\alpha$-particle peak intensity. A very good agreement of simulation and experimental data is observed in all cases, so that the described procedure can be reliably used to predict the detector behavior for any ${ }^{6} \mathrm{LiF}$-layer thickness. The only significant discrepancy is the intensity of the $2.73 \mathrm{MeV}$ tritium ion peak at the lower ${ }^{6} \mathrm{LiF}$-layer thickness. The simulated peak is in this case much narrower (and therefore more intense) because the broadening produced by the experimental noise and by the detector in-homogeneities is not taken into account in the simulation. Indeed, in this case the total number of counts (i.e., the area) below this peak is equal for the simulation and the experimental spectrum. We notice that although both the 2.73 $\mathrm{MeV}$ tritium ions and $2.07 \mathrm{MeV} \alpha$-particles peaks obviously exhibit a broadening with increasing ${ }^{6} \mathrm{LiF}-$ layer thickness, such a broadening is much more evident in the case of the $\alpha$-particles peak. In fact, the stopping power of ${ }^{6} \mathrm{LiF}$ for $2.07 \mathrm{MeV} \alpha$-particles is much higher than for 2.73 $\mathrm{MeV}$ tritium ions. The energy spread of the $\alpha$-particles reaching the detection layer is therefore higher and rapidly increasing with the ${ }^{6} \mathrm{LiF}$-layer thickness. In practice, a welldefined peak is only observed for the $0.45 \mu \mathrm{m}$ thickness.

From the PHA simulations, we can predict the full width at half-maximum (FWHM) versus LiF-layer thickness curves shown in Fig. 5 for both the $\alpha$-particles and the tritium ion peaks. While the tritium ion peaks maintain some

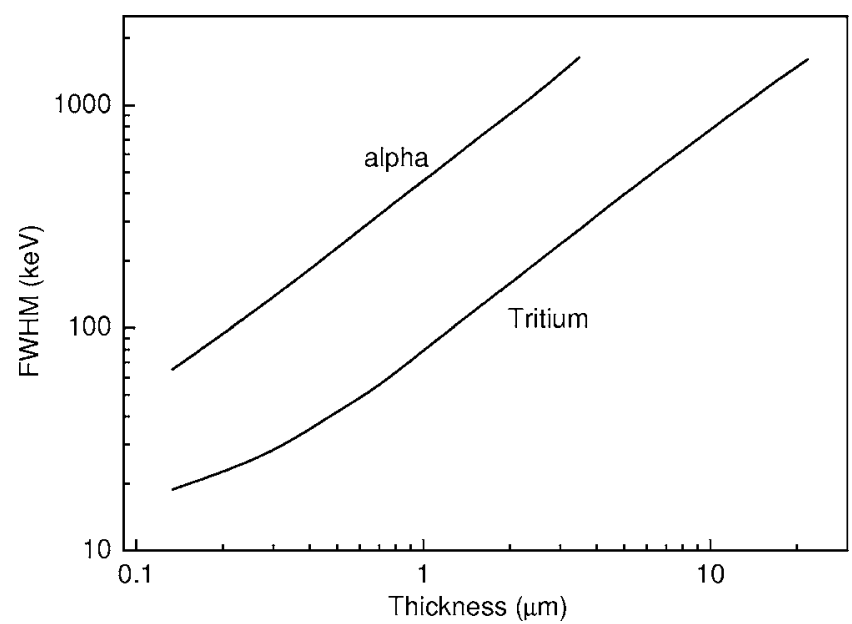

FIG. 5. Simulation of the FWHM of the $\alpha$ and tritium peaks as a function of the ${ }^{6} \mathrm{LiF}-$ layer thickness.

energy resolution up to thicknesses in the range of several micrometers, in the case of $\alpha$-particles, submicrometer thickness is mandatory to have a real peak.

When the detector is used as a neutron counter, an energy threshold, above which the signal is considered as a result of the detection of a thermal neutron, must be set. We have simulated the counting sensitivity of the detector (i.e., counts per incident neutrons) as a function of ${ }^{6} \mathrm{LiF}$-layer thickness. A thermal cross-section value of $950 \mathrm{~b}$ for the $(n, \alpha)$ reaction in ${ }^{6} \mathrm{Li}$ was adopted in the calculation. The results are shown in Fig. 6(a) for three different choices of the threshold: 2065,1000 , and $500 \mathrm{KeV}$, respectively. For the higher threshold, which is just above the $\alpha$-particles peak (see Fig. 4), only tritium ions can be detected, while in the other two cases both tritium and $\alpha$-particles are counted. In particular, the lower threshold is chosen just above the experimental low-energy signal increase. In all cases, an $\mathrm{Al}$ contact $100 \mathrm{~nm}$ thick is considered. The extremely limited absorption of neutrons in the ${ }^{6} \mathrm{LiF}$ layer is neglected, so that a uniform neutron density along the ${ }^{6} \mathrm{LiF}$ thickness is considered.

For the lower threshold values, the counting sensitivity monotonically increases with the ${ }^{6} \mathrm{LiF}$-layer thickness up to the maximum value considered in the simulation $(20 \mu \mathrm{m})$. For the $2065 \mathrm{KeV}$ threshold value, on the contrary, the counting sensitivity curve saturates for ${ }^{6} \mathrm{LiF}$-layer thickness higher than about $10 \mu \mathrm{m}$. Indeed, the $10 \mu \mathrm{m}$ length is the distance needed for the tritium ions to reduce their energy down to about 2.1 MeV so that tritium ions generated at distances higher than $10 \mu \mathrm{m}$ are not counted above this threshold. For this reason, any further increase in thickness could not add tritium ion counts, and a saturation of the counting sensitivity is observed.

For the 500 and $1000 \mathrm{keV}$ thresholds no saturation is observed, the distance needed to reduce the tritium energy below the threshold levels being larger than the maximum thickness $(20 \mu \mathrm{m})$ considered in the simulation. In Fig. 6(b), the sensitivity curve for the threshold value of $500 \mathrm{keV}$ is decomposed into the contributions due to $\alpha$-particles and tritium ions. In this case, the $\alpha$-particles contribution flattens 


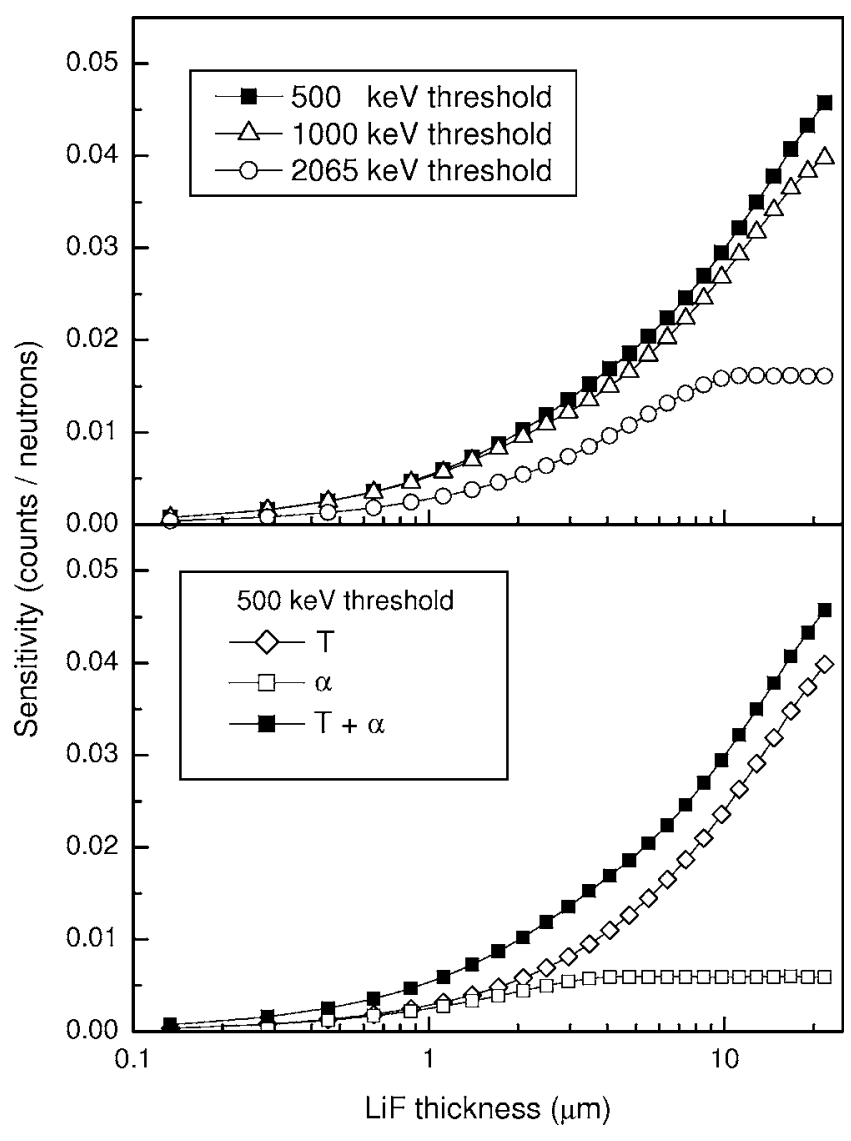

FIG. 6. Simulated counting sensitivity under thermal irradiation as a function of the ${ }^{6} \mathrm{LiF}$ thickness (a) for three different thresholds: 500, 1000, and $2065 \mathrm{keV}$, respectively. In (b), the sensitivity for the $500 \mathrm{keV}$ threshold is decomposed in the $\alpha$ and tritium contributions.

out when the ${ }^{6} \mathrm{LiF}$ layer becomes thicker than $4 \mu \mathrm{m}$. In fact, this is approximately the penetration depth of $\alpha$-particles in ${ }^{6} \mathrm{LiF}$, so that counts from $\alpha$-particles generated at a distance larger than $4 \mu \mathrm{m}$ are lost in the $\mathrm{LiF}$ layer. On the other hand, the tritium ions contribution increases steadily up to at least $20 \mu \mathrm{m}$, as does the total sensitivity.

\section{Fast neutrons}

High-energy neutrons have a great penetration depth. The presence of the ${ }^{6} \mathrm{LiF}$ layer has no effect on fast neutrons, which easily go across it and the active diamond layer too (the mean free path of $14.8 \mathrm{MeV}$ neutrons in diamond is about $3.8 \mathrm{~cm}$ ).

The expected PHA spectra were simulated according to the following procedure. The stopping power curve of $\alpha$-particles and ${ }^{9} \mathrm{Be}$ ions in diamond was approximated to Eq. (4) using the same procedure previously reported. Monte Carlo simulations were then performed by generating homogeneously inside the diamond layer $\alpha$-particle and ${ }^{9} \mathrm{Be}$ ions emission points. The distribution of the emission angle of the $\alpha$-particle is chosen according to the differential cross section of the reaction ${ }^{12} \mathrm{C}\left(n, \alpha_{0}\right){ }^{9} \mathrm{Be}$ reported in Ref. 10, while the emission angle of the corresponding ${ }^{9} \mathrm{Be}$ ions and the energy of both $\alpha$-particle and ${ }^{9} \mathrm{Be}$ ions are calculated from

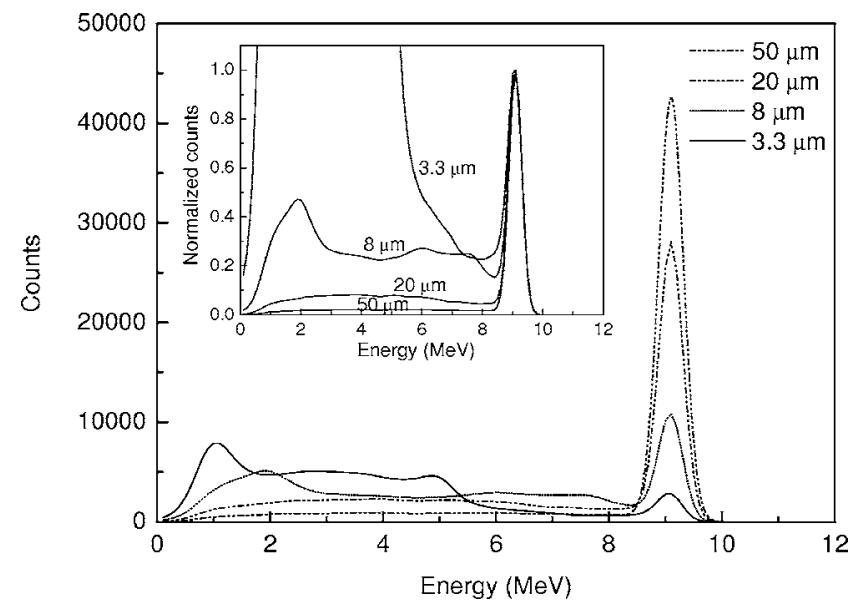

FIG. 7. Simulated PHA spectra under $14.8 \mathrm{MeV}$ neutron irradiation relative to the ${ }^{12} \mathrm{C}\left(n, \alpha_{0}\right){ }^{9} \mathrm{Be}$ peak for different intrinsic diamond thicknesses: $3.3,8$, 20 , and $50 \mu \mathrm{m}$, respectively. In the inset, the spectra normalized to the peak amplitude are reported.

the kinematic of the reaction. For each reaction, the energy released in the sensitive diamond layer by both $\alpha$-particles and ${ }^{9} \mathrm{Be}$ ions is finally calculated.

An interesting feature of fast neutron collection is the well-defined peak visible in Fig. 2 at about $9.1 \mathrm{MeV}$. The width of this peak is the same as that of the FNG neutron beam energy spread (about $0.5 \mathrm{MeV}$ ). In principle, a significant broadening of this peak would be expected at first glance since the detector thickness $(25 \mu \mathrm{m})$ is comparable with the penetration depth of the ${ }^{12} \mathrm{C}(n, \alpha){ }^{9} \mathrm{Be}$ reaction products in diamond, i.e., from 3 to $30 \mu \mathrm{m}$ for $\alpha$-particles and from 0.5 to $6 \mu \mathrm{m}$ for Be ions, depending on their energy, which is related to the angle of generation. It turns out, therefore, that a significant part of the $\alpha$-particles and ${ }^{9} \mathrm{Be}$ ions does not lose all its energy inside the active layer, and this should induce a large peak broadening. However, the distribution of the energy released by these $\alpha$-particles and $\mathrm{Be}$ ions inside the detector turns out to be rather flat below the 9.1 MeV peak. A decrease in the detector thickness has the effect of removing some counts from the $9.1 \mathrm{MeV}$ peak and distributing them almost uniformly in the lower energy background, therefore causing no noticeable broadening.

The effect of this process is visualized in Fig. 7, where the simulated spectrum calculated for monochromatic 14.8 $\mathrm{MeV}$ neutrons is convoluted with a $0.5 \mathrm{MeV}$ FWHM Gaussian function representing the energy spread of the beam. The result of the convolution, plotted in Fig. 7 for several values of the detector thickness, is a peak at $9.1 \mathrm{MeV}, 0.5 \mathrm{MeV}$ wide, with an almost flat low-energy background. The only effect of reducing the detector thickness is a gradual decrease of the peak height and a corresponding moderate increase of the background level. Only for very thin detectors are the peak and background levels comparable. Therefore, good energy resolution in the detection of highly penetrating neutrons can be obtained with our detectors even for very low thickness values, with a great advantage in terms of fabrication cost. On the other hand, the higher the detector thickness, the higher its sensitivity. This is due to both the higher number of reactions occurring in thicker samples and to 


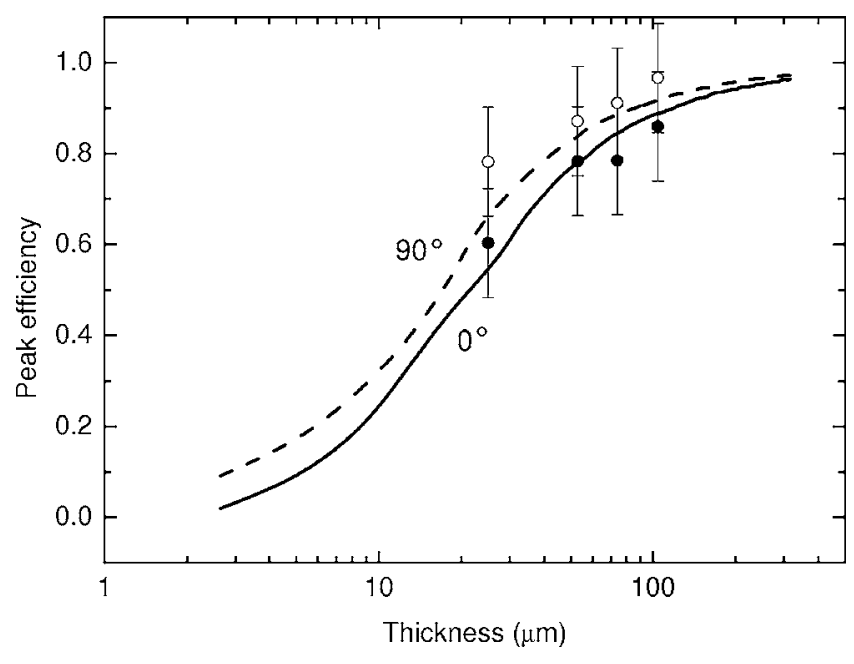

FIG. 8. Peak efficiency as a function of the intrinsic diamond layer thickness under $14.8 \mathrm{MeV}$ neutron irradiation at $0^{\circ}$ (full line) and $90^{\circ}$ (dashed line) incidence angle. The circles are experimental data at $0^{\circ}$ (full circles) and $90^{\circ}$ (open circles) incidence angle, respectively.

boundary effects limiting the complete collection of the reaction products in thin samples. To better analyze this aspect, we define the peak efficiency as the ratio between the counted $\mathrm{Be}+\alpha$ particles inside the $9.1 \mathrm{MeV}{ }^{12} \mathrm{C}(n, \alpha){ }^{9} \mathrm{Be}$ peak and the number of ${ }^{12} \mathrm{C}(n, \alpha){ }^{9} \mathrm{Be}$ reactions. The counting sensitivity per unit area can be derived easily from the peak efficiency by multiplying it by the cross section of the ${ }^{12} \mathrm{C}(n, \alpha){ }^{9} \mathrm{Be}$ reaction, the detector thickness, and the ${ }^{12} \mathrm{C}$ atomic concentration.

In Fig. 8, a simulation of the peak efficiency as a function of the active diamond layer thickness is reported. The lower curve (full line) is the simulation for the $0^{\circ}$ incidence angle of the neutron beam with respect to the detector surface normal (i.e., the neutron beam perpendicular to the sample surface); the upper curve (dashed line) is for the $90^{\circ}$ incidence angle.

In both cases, optimal collection takes place only for thickness much higher than the penetration depths of the reaction products. It is also evident from Fig. 8 that the calculated peak efficiency is always higher in the case of $90^{\circ}$ incidence angle. This is due to the kinematics of the reaction, which produces more energetic reaction products toward the irradiation direction. These more penetrating ions can easily escape from the opposite side of the detector reducing the peak efficiency when the detector is set at $0^{\circ}$.

A comparison with experimental data acquired with four different detectors $(25,53,74$, and $104 \mu \mathrm{m}$ thick, respectively) irradiated both in the $0^{\circ}$ direction (full circle) and in the $90^{\circ}$ direction (open circles) is also reported in Fig. 8. The measured peak efficiencies were extracted from the experimental PHA spectra by calculating the ratio between the counts in the ${ }^{12} \mathrm{C}(n, \alpha){ }^{9} \mathrm{Be}$ peak and the number of
${ }^{12} \mathrm{C}(n, \alpha){ }^{9} \mathrm{Be}$ reactions, where the latter is given by the total count number multiplied by the cross section of the ${ }^{12} \mathrm{C}(n, \alpha){ }^{9} \mathrm{Be}$ reaction $(72 \mathrm{mb})$ and divided by the total cross section of $14.8 \mathrm{MeV}$ neutrons in carbon (1.2 b).

The obtained experimental points are in good agreement with the simulation results, demonstrating that this analysis allows to calculate in advance the detector behavior for any diamond layer thickness.

\section{CONCLUSION}

We have presented a detailed study of the physics underneath the working principles of a detector capable of simultaneously detecting thermal and fast neutrons. The detectors are fabricated from CVD single-crystal diamond films using a $p$-type/intrinsic/metal configuration. Fast neutrons are detected directly in the bulk of the detector through the ${ }^{12} \mathrm{C}(n, \alpha){ }^{9} \mathrm{Be}$ reaction, while thermal ones are detected through the ${ }^{6} \mathrm{Li}(n, \alpha) T$ reaction taking place in a thin ${ }^{6} \mathrm{LiF}$ deposited on top of the detector. An analysis of the nuclear reaction kinematics allows us to calculate the resolution and sensitivity of the detector for both fast and thermal neutrons, given the thickness of the diamond detector and of the ${ }^{6} \mathrm{LiF}$ layer. The results show that good resolution for thermal neutrons requires thin ${ }^{6} \mathrm{LiF}$ layers, in a trade-off with detection sensitivity. On the other hand, sensitivity and energy resolution for fast neutrons increase with the detector thickness, in a trade-off with cost. Surprisingly, however, good resolution is obtained even when the detector thickness is lower than the range of the $\alpha$-particles detected.

These results are in good agreement with experimental data, showing that this analysis allows us to calculate in advance the best choice of detector and ${ }^{6} \mathrm{LiF}$-layer thickness for any given application.

${ }^{1}$ J. H. Kaneko, T. Tanaka, T. Imai, Y. Tanimura, M. Katagiri, T. Nishitani, H. Takeuchi, T. Sawamura, and T. Iida, Nucl. Instrum. Methods Phys. Res. A 505, 187 (2003)

${ }^{2}$ G. J. Schmid, J. A. Koch, R. A. Lerche, and M. J. Moran, Nucl. Instrum. Methods Phys. Res. A 527, 554 (2004).

${ }^{3}$ C. Mer, M. Pomorski, P. Bergonzo, D. Tromson, M. Rebisz, T. Domenech, J. C. Vuillemin, F. Foulon, M. Nesladek, O. A. Williams, and R. B. Jackman, Diamond Relat. Mater. 13, 791 (2004).

${ }^{4}$ P. Lardon, C. Mer, P. Delacour, B. Barzin, D. Tromson, S. Normand, M. Nesladek, F. Foulon, and P. Bergonzo, Diamond Relat. Mater. 15, 815 (2006).

${ }^{5}$ M. Marinelli, E. Milani, G. Prestopino, M. Scoccia, A. Tucciarone, G. Verona-Rinati, M. Angelone, M. Pillon, and D. Lattanzi, Appl. Phys. Lett. 89, 143509 (2006)

${ }^{6}$ M. Marinelli, E. Milani, G. Prestopino, A. Tucciarone, G. Verona-Rinati, M. Angelone, D. Lattanzi, and M. Pillon, Appl. Phys. Lett. 90, 183509 (2007).

${ }^{7}$ M. Martone, M. Angelone, and M. Pillon, J. Nucl. Mater. 212-215, 1661 (1994).

${ }^{8}$ JANIS-1, 2, Java-based nuclear data display program, NEA OECD (2004) (http://www.nea.fr/Janis).

${ }^{9}$ J. F. Ziegler, Nucl. Instrum. Methods Phys. Res. B 219-220, 1027 (2004).

${ }^{10}$ R. C. Haight, S. M. Grimes, R. G. Johnson, and H. H. Barschall, Nucl. Sci. Eng. 87, 41 (1984). 